\title{
The scenario-based approach for control of multi-object dynamic system motion
}

\author{
O. Tachinina \\ National Aviation University \\ Kyiv, Ukraine \\ tachinina@rambler.ru \\ O. Lysenko \\ National Technical University of Ukraine "KPI", \\ Kyiv, Ukraine \\ lysenko.a.i.1952@gmail.com
}

\author{
S. Chumachenko \\ Ukrainian Scientific Research Institute \\ of Civil Protection, \\ Kyiv, Ukraine \\ s_chum@ukr.net
}

\begin{abstract}
This paper proposes a scenario-based approach for control of multi-object dynamic system motion for the cases when the relative position of the objects relative each to other plays the primary role.
\end{abstract}

Keywords: control, multi-object dynamic system

\section{INTRODUCTION}

The interest for pilotless aviation is increased at almost all developed countries recent years. Due to the revolutionary development of a number of technical areas the unmanned aerial vehicles (UAVs) have become an effective tool to solve the tasks of wide range. The UAV had mostly military designation up to the present, however new civil tasks, which can be resolved successfully by UAVs are occurred. Thus UAVs gain popularity as an easy and inexpensive tool for research, reconnaissance, aerial survey. In Ukraine, as well worldwide the works for implementation the UAV are being developed in the environmental and agricultural areas, as well as for various tasks of terrain monitoring at emergency situation areas. [5].

\section{PROBLEM STATEMENT}

In practice, the single UAV is used usually, in turn, it leads to low efficiency of task performance, especially concerning large areas. These limitations are caused by limited coverage of the UAV's sensory systems.

The advantage of using UAVs group becomes evident in the tasks where parallelization of difficult task for several vehicles is possible, e.g. in the task of monitoring large areas in a short time, which is a problem when using a single vehicle.

To achieve this goal the UAV group should act as whole object and the actions of each individual UAV should be directed to the largest group effect. For this purpose it is proposed the UAV group performing a single goal to consider as the multi-object dynamic system (MDS)

The MDS is interpreted as a set of objects (UAVs) combined in the physical sense of the task.

With many advantages of UAV group use there are a number of problems arising from the need to organize its collective behavior for solving common technological task. One of these problems is to create algorithms for the organization of effective interaction between the UAV at all hierarchy levels at which the

ultimate goal is achieved (or target condition) in minimum time and at minimal cost.

The aim of this work is to apply the scenario-based approach to the MDS control.

\section{THE SCENARIO-BASED APPROACH FOR CONTROL OF MULTI-OBJECT DYNAMIC SYSTEM MOTION}

In this paper four-engined aircraft with vertical thrust vector or quadrocopters are considered as the objects of the MDS. Since quadrocopters are unstable objects of control [8], the main problem arising when creating the multi-object control strategy is to achieve sustainable movement of MDS to a specific point in a space.

The quadrocopter is a type of aircraft with four rotors, driving by four motors at rate of rotation $\Omega 1$, $\Omega 2, \Omega 3, \Omega 4$, installed in the UAV center of mass $M$ on two intersecting crosswise metal beams, rotating in opposite directions diagonally (fig. 1).

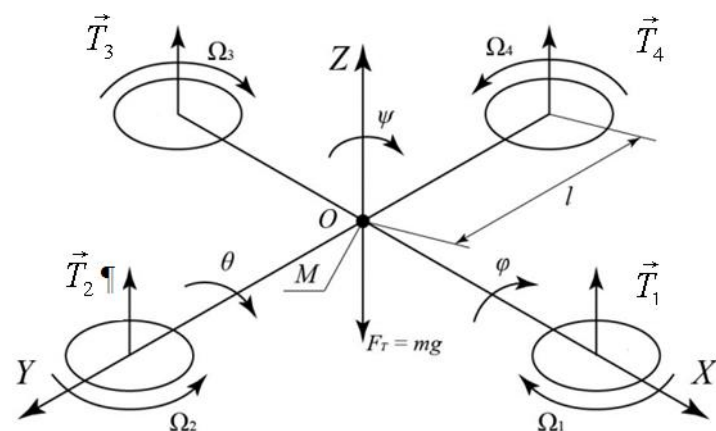

Fig. 1. Quadrocopter's body-fixed reference system

To synthesize the top-level control strategies it is necessary to build a mathematical model of the quadrocopter considered as a solid body. Suppose that the quadrocopters contained in the group have the same design and inertial specifications, with the center of mass of each robot is located at the intersection of the beams, on which the rotors are secured [6]. The quadrocopters group motion mode will be described by the following differential equations [6]: 


$$
\begin{aligned}
& \dot{x}^{k}=V_{x}{ }^{k} ; \dot{y}^{k}=V_{y}^{k} ; \dot{z}^{k}=V_{z}^{k} \\
& \dot{V}_{x}^{k}=\left(\sin \psi^{k} \sin \varphi^{k}+\cos \psi^{k} \sin \theta^{k} \cos \varphi^{k}\right) \frac{U_{1}^{k}}{m} ; \\
& \dot{V}_{y}{ }^{\mathrm{k}}=\left(-\cos \psi^{\mathrm{k}} \sin \varphi^{\mathrm{k}}+\sin \psi^{\mathrm{k}} \sin \theta^{\mathrm{k}} \cos \varphi^{\mathrm{k}}\right) \frac{\mathrm{U}_{1}^{\mathrm{k}}}{\mathrm{m}} ;(3) \\
& \dot{V}_{z}^{k}=\left(\cos \theta^{k} \cos \varphi^{k}\right) U_{1}^{k} / m-g \\
& \dot{\theta}^{k}=\omega_{\theta}^{k} ; \dot{\varphi}^{k}=\omega_{\varphi}^{k} ; \dot{\psi}^{k}=\omega_{\psi}^{k} ; \\
& \dot{\omega}_{\varphi}^{k}=\frac{\left(I_{y y}-I_{z z}\right) \omega_{\theta}^{k} \omega_{\psi}^{k}-J_{T P} \omega_{\theta}^{k} \Omega^{k}+U_{2}^{k}}{I_{x x}} \\
& \dot{\omega}_{\theta}^{k}=\frac{\left(I_{z z}-I_{x x}\right) \omega_{\varphi}^{k} \omega_{\psi}^{k}+J_{T P} \omega_{\varphi}^{k} \Omega^{k}+U_{3}^{k}}{I_{y y}} \\
& \dot{\omega}_{\psi}^{k}=\frac{\left(I_{x x}-I_{y y}\right) \omega_{\varphi}^{k} \omega_{\theta}^{k}+U_{4}^{k}}{I_{z z}}
\end{aligned}
$$

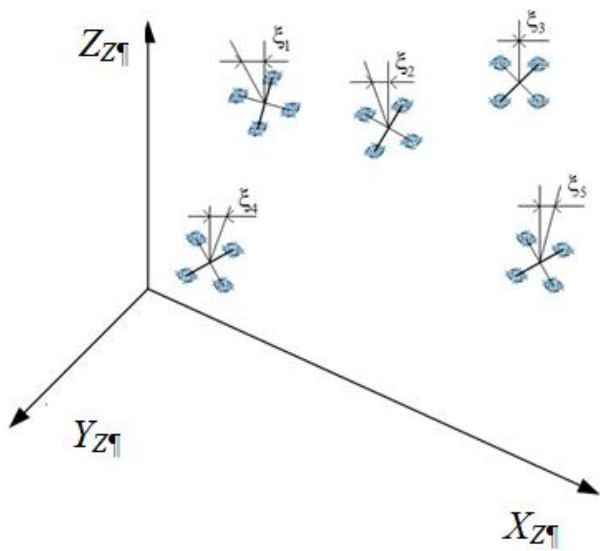

a)

Fig.2. Allocation of UAVs: a) arbitrary, б) specified.

$$
\begin{aligned}
& \Omega_{1}^{k}=\sqrt{\frac{1}{4 b} U_{1}^{k}-\frac{1}{2 b l} U_{3}^{k}-\frac{1}{4 d} U_{4}^{k}} ; \\
& \Omega_{2}^{k}=\sqrt{\frac{1}{4 b} U_{1}^{k}-\frac{1}{2 b l} U_{2}^{k}+\frac{1}{4 d} U_{4}^{k}} ; \\
& \Omega_{3}^{k}=\sqrt{\frac{1}{4 b} U_{1}^{k}+\frac{1}{2 b l} U_{3}^{k}-\frac{1}{4 d} U_{4}^{k}} ; \\
& \Omega_{4}^{k}=\sqrt{\frac{1}{4 b} U_{1}^{k}+\frac{1}{2 b l} U_{2}^{k}+\frac{1}{4 d} U_{4}^{k}} ; \\
& \Omega^{k}=-\Omega_{1}^{k}+\Omega_{2}^{k}-\Omega_{3}^{k}+\Omega_{4}^{k} .
\end{aligned}
$$

where $l$ - distance between quadrocopter's center and rotor's center, $b$ - и $d$ - thrust aerodynamic component and coefficient of air resistance.

One task of the group control is to build the UAVs flight formation for the ordered flight from one location to another. Another task is the distribution of a defined configuration in the space what the UAVs group will arrange for the specific task.

where $x^{k}, y^{k}, z^{k}$ - center of mass coordinates of $k$ quadrocopter of group; $V_{\mathrm{x}}{ }^{k}, V_{\mathrm{y}}{ }^{k}, V_{\mathrm{z}}{ }^{k}$ - projections of $k$ - quadrocopter linear velocity; $\theta^{k}, \varphi^{k}, \psi^{k}-k$ quadrocopter angle of pitch, roll angle and yaw angle; $\omega_{\theta}^{k}-k$ - UAV pitch rate; $\omega_{\varphi}^{k}-k$ - UAV rate of roll, $\omega_{\psi}^{k}$ $k$ - UAV rate of yaw; $U_{1}{ }^{k}, U_{2}{ }^{k}, U_{3}{ }^{k}, U_{4}{ }^{k}-k$ - UAV control channels; $\Omega^{k}-k$ - UAV overall speed of four rotors; $m$ - quadrocopter mass; $I_{x x}, I_{y y}, I_{z z}-$ moments of inertia around the $X_{z}, Y_{z}$ и $Z_{z}$ axis; $I_{T P}$ total rotational inertia moment around the rotor axis.

The equations of relation of control channels $U_{1}{ }^{k}, \quad U_{2}{ }^{k}, U_{3}{ }^{k}, \quad U_{4}{ }^{k}$ with rotor rotation rates $\Omega_{1}{ }^{k}, \Omega_{2}{ }^{k}, \Omega_{3}{ }^{k}, \Omega_{4}{ }^{k}$ are of the form of:

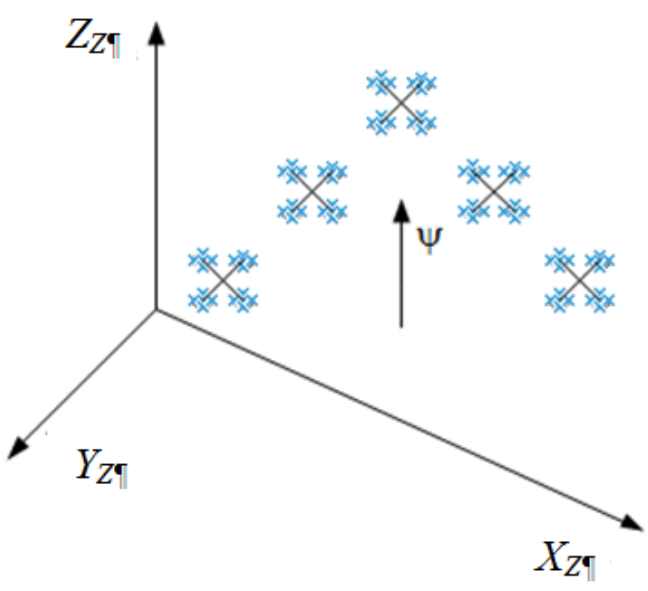

б)

Suppose the group $\boldsymbol{R}$ of $\boldsymbol{n}$ quadrocopters $\mathrm{r}_{\mathrm{k}} \in \boldsymbol{R}(k=\overline{l, n})$ flies in the air at arbitrary position at some time (fig. 2.1). Also, UAVs can be different from each other by flight direction (heading) and speed.

In order that the quadrocopters group arranges some configuration in space it is necessary to distribute the UAVs group to positions of the flight formation.

The flight formation is intended for consolidated, coordinated flight of UAVs group, as well as for the collective execution of the assigned task. The flight formation is specified as the coordinates of the target points in the absolute fixed-in-the-earth coordinate system.

The task of transition to the selected flight formation is to order the UAVs group from an arbitrary state in a specific state with the necessary variables $x^{0} \rightarrow x^{\mathrm{f}}$. The state variables of $k$ quadrocopter are its coordinates in space $x^{k}$, 
$y^{k}, z^{k}$, its current speed, acceleration, angles of roll $\varphi^{k}$, pitch $\theta^{k}$ and yaw $\psi^{k}$ and remaining board energy reserves.

In this case the UAVs may be arranged in the space arbitrarily, so the main goal that they were located in a limited area and have a similar speed at distribution time and direction vector of speed was situated within the predetermined heading $\psi^{k} \pm \Delta \xi \leq \psi$, where $\psi^{k}$ - heading of $k$ - UAV; $\psi$ specified heading of the flight formation; $\Delta \xi$ - some angle of $k$ - UAV heading permissible deviation relative to heading of the flight formation $\psi$.

Deviation of $k$ - UAV heading $\psi^{k}$ from $\psi$ is denoted

as $\xi_{\mathrm{k}} \leq \Delta \xi(k=\overline{l, n})$, where $n$ - number of UAVs in the group (fig.2).

When the MDS transits to the specified flight formation, it is important how to set the flight formation, what "relationship" should be between the flight formation and the MDS before distribution, at the time of distribution and after distribution, when the UAVs are beginning to arrange a specified locations.

To arrange the UAVs in the specified flight formation we will use the scenario approach based on the criteria of smallest total movement.

This approach will provide the minimization of the total movement of the UAVs from some arbitrary

coordinates $\quad x_{k}^{0}=\left\{x_{1}^{0}, x_{2}^{0} \ldots, x_{n}^{0}\right\} \quad(k=\overline{1, n}) \quad$ in required states described by vector $x_{k}^{f}=\left\{x_{1}^{f}, x_{2}^{f} \ldots, x_{n}^{f}\right\}(k=\overline{1, n})$.

We consider a scenario-based approach to control the movement of MDS in the flight formation. The kernel of this method is to find such combination of "UAV - target" in order to the lowest result will reached at summation all distances received after the distribution. This is known as "classical assignment problem" [6].

To find the solution the relative position of quadrocopters at the group is described by matrix

$$
\mathrm{D}_{0}=\left|\begin{array}{ccccc}
0 & \mathrm{~d}_{12} & \mathrm{~d}_{13} & \ldots & \mathrm{d}_{1 \mathrm{n}} \\
\mathrm{d}_{21} & 0 & \mathrm{~d}_{23} & \ldots & \mathrm{d}_{2 \mathrm{n}} \\
\mathrm{d}_{31} & \mathrm{~d}_{32} & 0 & \ldots & \mathrm{d}_{3 \mathrm{n}} \\
\ldots & \ldots & \ldots & 0 & \mathrm{~d}_{\mathrm{n} 1 \mathrm{n}} \\
\ldots & \ldots & \ldots & \ldots & 0
\end{array}\right|
$$

where each element $d_{i j}$ - matrix $D_{0}$ represents the distance between quadrocopters $r_{i}$ and $r_{j}$ in the current instant of time.
Each quadrocopter $r_{i} \in \boldsymbol{R}$ have available the data of eigenstate $x_{i}^{0}$, as well as information of distances $\quad \mathrm{d}_{\mathrm{ij}}(i, \mathrm{j}=\overline{l, n}, \mathrm{i} \neq \mathrm{j}) \quad$ between quadrocopter $r_{i}$ and other quadrocopters $r_{j} \in \boldsymbol{R}$ $(\mathrm{j}=\overline{1, n}, \mathrm{j} \neq \mathrm{i})$.

Each quadrocopter $r_{i} \in \quad \boldsymbol{R}$ can modify coordinates $x^{k}, y^{k}, z^{k}$ of own position in space, depending on the input controls $U^{k}$ in accordance with the mathematical model (9-13).

The target formation position (target formation) of quadrocopters group is a set of $Q$ target positions $q_{\mu} \in Q \quad(\mu=\overline{1, N})$ of separate quadrocopters. Each target position of quadrocopters $q_{\mu} \in Q$ is characterized by $p_{\mu}\left(x_{\mu}, y_{\mu}, z_{\mu}\right)(\mu=\overline{1, N})$.

The target position of group is specified by matrix:

$$
\mathrm{D}_{f}=\left|\begin{array}{ccccc}
0 & \mathrm{~d}_{12} & \mathrm{~d}_{13} & \ldots & \mathrm{d}_{1 \mathrm{n}} \\
\mathrm{d}_{21} & 0 & \mathrm{~d}_{23} & \ldots & \mathrm{d}_{2 \mathrm{n}} \\
\mathrm{d}_{31} & \mathrm{~d}_{32} & 0 & \ldots & \mathrm{d}_{3 \mathrm{n}} \\
\ldots & \ldots & \ldots & 0 & \mathrm{~d}_{\mathrm{n} 1 \mathrm{n}} \\
\ldots & \ldots & \ldots & \ldots & 0
\end{array}\right|,
$$

where each element $\mathrm{d}_{\mathrm{ij}}$ of matrix $\mathrm{D}_{f}$ represents a distance between the coordinates $p_{i}$ и $p_{j}$ of target position $q_{\mathrm{i}}$ и $q_{\mathrm{j}}$ in the required formation.

The formation task is to define such sequence of controls (control vector function) $U=\left\lfloor U_{1}, \mathrm{U}_{2}, \ldots, U_{N}\right\rfloor$ which implementation leads the group of quadrocopters from the starting position, specified matrix of distances $\mathrm{D}_{0}$ in proper formation position specified by matrix $\mathrm{D}_{f}$ at minimum time.

On the basis of this matrix the required configuration of the allocation is searched. The scenario-based approach is used to allocate the UAVs group on target points of the UAVs flight formation with the smallest total value of path lengths.

Distance matrix $D_{f}(\mu \times n)$ serves as a basis, where $n$ - the number of UAVs in the group, $\mu$ - the number of target points in the flight formation, configuration $n=\mu$. Values of matrix $D_{f \rightarrow} q_{i j}$ - is the shortest distance between $\mathrm{j}$ - UAV and $i$ - target point in the flight formation configuration. 


$$
q_{i j}=\sqrt{\left(x_{i}-x_{j}\right)^{2}+\left(y_{i}-y_{j}\right)^{2}+\left(z_{i}-z_{j}\right)^{2}},
$$

where $x_{i}, y_{i}, z_{i}$ - coordinates of $i$ - target point in the flight formation configuration; $\mathrm{x}_{\mathrm{j}}, \mathrm{y}_{\mathrm{j}}, \mathrm{z}_{\mathrm{j}}$ - current position of $j$ - UAV.

To provide this approach it is necessary to determine the distribution center of flight formation. For this purpose, the center of mass calculation equation is applied:

$$
L_{c}=\frac{\sum_{i=n}^{n} \vec{r}_{i} \cdot m_{i}}{\sum_{i=n}^{n} m_{i}},
$$

where $\vec{r}_{i}$ - radius vector of $i$ - target point of UAV in flight formation configuration, $m_{i}$ - mass analog, in this instance is a factor, which value changing allows to influence the position of "flight formation center of mass" regarding the $i$ - target point. In this case, it is assumed as one for all UAVs, then formula (14) takes the form:

$$
L_{c}=\frac{\sum_{i=n}^{n} \vec{r}_{i}}{N}
$$

The next step is to combine the centers of mass of specified configuration of UAVs flight formation (fig. 2,b) and UAVs group (fig. 2, a) along two axes $X_{3}$ and $Y_{3}$ as well along axis $Z_{3}$ in the direction of set heading $\psi$.

The scenario-based approach will consist of two stages.

First stage. The extreme-point solution is formed at the first stage as follows.

According to available matrix $D_{f}$ minimum estimation $\xi_{j}=\min \left(q_{i j}\right) \quad(i=\overline{1, m})$ is selected for each $\mathrm{j}$ - UAV starting with $j=1$. After selecting the corresponding row $i$ will no longer take part in the formation of the extreme-point solution. Further, such this procedure is performed for $\mathrm{j}=2$ and so on. This method of setting the extreme-point solution will reduce the number of steps to improve the generalized plan (algorithm) in the future. Once the extreme-point solution is formed, proceed to the next step.

Second stage. At this stage, the extreme-point solution for the pair of $\mathrm{j}$ - and $k$ - UAVs is optimized. The lowest distance value is retrieved relative to the extreme-point solution for the first UAV in the first column of the matrix $\mathrm{D}_{\mathrm{f}}$. If such is found in $n$-row, the value of the other $k$ - UAV standing on $n$ - row, should jump to the row where there was value of first $\mathrm{UAV}$, thus the transposition improve overall solution. If there is no improvement, the transposition is not fixed and the current status of the allocation remains in the former state.

Next, the same procedure is performed for the second UAV, where the smallest value of the distance is retrieved and the initial solution is optimized in the same manner. This procedure is performed for other UAVs $(\mathrm{j}=\overline{1, \mathrm{n}})$ (Fig.3).

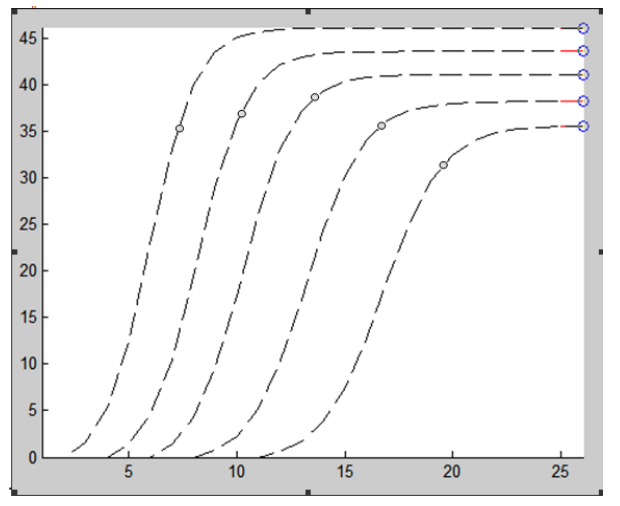

Fig.3. The UAVs group flight paths to the target configuration

The condition for the end of the UAVs allocation algorithm to the target point in the flight formation is absence of new transpositions in an iterative optimization of the solution.

\section{CONCLUSIONS}

This paper proposes the scenario-based approach to control the multi-object dynamic systems motion for the cases where the relative position of the objects relative each to other plays a primary role.

The proposed approach will allow generating the required configuration of UAV flight system formation at minimum evolutions and intersections on the planned paths, as well provide ample opportunities for MDS practical application to search for fires, pollution, etc., mapping, search for people on land and water, use in military service.

\section{REFERENCES}

[1] G. Veselov. A. Sklyarov. S. Sklyarov. Synergetic approach to control unmanned aircraft. Proceedings of the SFU. Technical science. - 2013. - № 5 (142). - P. 65-70.

[2] A Kalyaev. V Makarov. I. Lokhin and others. Intelligent robots. Under the general editorship. E. Yurevich. Mechanical Engineering, 2007. - 360 p.

[3] I. Kalyaev. A. Haiduk. S. Kapustyan. Methods and models of collective control in groups of robots. - M.: FIZMATLIT, 2009. - $280 \mathrm{p}$.

[4] I. Kalyaev. S. Kapustyan. A. Haiduk. Rapredelennye Selforganizing control teams of intelligent robots that are based on the network model . Managing large systems: Sat. works. - 2010.- № 30 (1) -- P.605-639.

[5] O. Lysenko. O. Tachinina. The mathematical formulation of the optimization problem of motion group flying robots based unmanned aerial vehicles. Scientific Bulletin of the Academy of Municipal Management. - K .: AMM, 2014. Vol. 1 (7). - P. 83-100

[6] Tommaso Bresciani. Modeling, identification and control of a quadrotor helicopter. - Lund: Master thesis, 2008. - 170 p. 